\title{
Estratégias usadas para a prevenção e tratamento da Síndrome de Burnout
}

\author{
Strategies used for the prevention and treatment of Burnout Syndrome \\ Estrategias utilizadas para la prevención y el tratamiento del síndrome de Burnout
}

Recebido: 31/03/2021 | Revisado: 10/04/2021 | Aceito: 16/04/2021 | Publicado: 01/05/2021

\author{
Suiane dos Santos Fialho De Lima \\ ORCID: https://orcid.org/0000-0001-7596-9420 \\ Universidade Federal do Pará, Brasil \\ E-mail: suianefialholima@ @otmail.com \\ Maria Fâni Dolabela \\ ORCID: https://orcid.org/0000-0003-0804-5804 \\ Universidade Federal do Pará, Brasil \\ E-mail: fani@ufpa.br; fanidolabela20@gmail.com
}

\begin{abstract}
Resumo
Objetivo: O presente estudo realizou uma revisão integrativa com objetivo de identificar estratégias para a prevenção e tratamento da síndrome de Burnout, bem como analisar a resposta desta estratégia. Metodologia: A pesquisa foi realizada no portal de periódicos, sendo utilizado o seguinte descritor-treatment and burnout., sendo 73 resumos selecionados para a leitura e ao final foram incluídos 18 artigos. Resultados: Para prevenção e tratamento da síndrome de Burnout é importante alterações na organização, na postura da equipe e o fortalecimento de estratégias de enfrentamento individual. Entretanto, a maioria dos estudos selecionados trabalham, principalmente, no campo do indivíduo. Para prevenção, estratégias de autocuidado, reconhecimento precoce dos sinais pelos colegas, supervisão clínica, modificações dos fatores estressores, treinamentos que visem ajudar o indivíduo lidar com os estressores, bem como alterações organizações parecem ser importantes. Para o tratamento do esgotamento a terapia cognitiva comportamental, Competência emocional, Psicoterapia em grupo ou individuais, treinamento de competências, coesão entre os pares, luz forte, método holístico, acupuntura, arte terapia e cursos de treinamento que visem mudanças de atitudes contribuíram para melhoria da síndrome. Ressalta-se que é importante avaliara, além dos aspectos laborais, o impacto da vida pessoal no adoecimento. Conclusão: Em síntese, é importante que a equipe conheça a síndrome, seja traçadas estratégias para prevenção e reconhecimento, bem como seja devolvido estratégias para a redução dos fatores estressores e fortalecimento das relações pessoais.
\end{abstract}

Palavras-chave: Burnout; Prevenção; Tratamento.

\begin{abstract}
Objective: This study carried out an integrative review in order to identify the prevention and treatment of Burnout syndrome, as well as to analyze the response of this strategy. Methodology: A search was carried out on the journals portal, using the following descriptor-treatment and burnout., 73 abstracts were selected for reading and at the end 18 articles were included. Results: To prevent and treat Burnout syndrome, it is important to change the organization, the team's posture and the strengthening of individual coping. However, most studies select, mainly, in the field of the individual. For prevention, self-care strategy, early recognition of signs by colleagues, clinical supervision, modifications of stressors, training to help the individual deal with stressors, as well as changes, alterations seem to be important. For the treatment of exhaustion, cognitive behavioral therapy, emotional competence, group or individual psychotherapy, skills training, cohesion between peers, strong light, holistic method, acupuncture, art therapy and training courses aimed at changing attitudes contributed to improvement. of the syndrome. It is noteworthy that it is important to assess, in addition to work aspects, the impact of personal life on illness. Conclusion: In summary, it is important that the team knows the syndrome, that it is designed for prevention and recognition, as well as that it be returned to reduce stressors and strengthen personal relationships.
\end{abstract}

Keywords: Burnout; Prevention; Treatment.

\section{Resumen}

Objetivo: Este estudio realizó una revisión integradora con el fin de identificar la prevención y tratamiento del síndrome de Burnout, así como analizar la respuesta de esta estrategia. Metodología: Se realizó una búsqueda en el portal de revistas, utilizando el siguiente descriptor-tratamiento y burnout. Se seleccionaron 73 resúmenes para lectura y al final se incluyeron 18 artículos. Resultados: Para prevenir y tratar el síndrome de Burnout es importante cambiar la organización, la actitud del equipo y el fortalecimiento del afrontamiento individual. Sin embargo, la mayoría de estudios seleccionan, principalmente, en el campo del individuo. Para la prevención, la estrategia de autocuidado, el reconocimiento temprano de los signos por parte de los colegas, la supervisión clínica, las modificaciones de los factores estresantes, el entrenamiento para ayudar al individuo a lidiar con los factores estresantes, así como los 
cambios, las alteraciones parecen ser importantes. Para el tratamiento del agotamiento, la terapia cognitivoconductual, la competencia emocional, la psicoterapia grupal o individual, el entrenamiento de habilidades, la cohesión entre pares, la luz fuerte, el método holístico, la acupuntura, la arteterapia y los cursos de capacitación dirigidos a cambiar actitudes contribuyeron a la mejora del síndrome. Es de destacar que es importante evaluar, además de los aspectos laborales, el impacto de la vida personal sobre la enfermedad. Conclusión En resumen, es importante que el equipo conozca el síndrome, que sea perfilado para su prevención y reconocimiento, así como que sea devuelto para reducir los estresores y fortalecer las relaciones personales.

Palabras clave: Burnout: Prevención; Tratamiento.

\section{Introdução}

A Síndrome de Burnout é um fenômeno ocupacional caracterizado pelo esgotamento, que resulta do estresse crônico no local de trabalho. Esta se caracteriza em três dimensões: sentimentos de exaustão ou exaustão de energia, aumento da distância mental do trabalho, ou sentimentos de negativismo relacionados ao trabalho e eficácia profissional reduzida (Kirby, et al., 2020). Outras doenças podem afetar o profissional, como a depressão e ansiedade, porém a Síndrome de Burnout se diferencia desata doenças devido envolver uma estafa física, mental e emocional (Teixeira, et al., 2010; Cândido \& Rocha De Souza, 2017).

Em termos conceituais, a exaustão emocional, presente nesta síndrome, é caracterizada pela diminuição ou falta de energia e sensação de esgotamento de recursos. Enquanto que, a despersonalização se manifesta como uma falta de sensibilidade emocional levando ao distanciamento, a impessoalidade, a desmotivação, a alienação e egoísmo. Mesmo que as pessoas achem que o profissional é bem sucedido, este tem uma autoavaliação negativa em relação suas atividades laborais, isso acarreta um sentimento de inadequação pessoal e profissional (Rissardo \& Gasparino, 2013; Sé, et al., 2020).

Vários profissionais podem apresentar esta síndrome, entretanto, ela é muito frequente entre os profissionais da saúde (Sé, et al., 2020) e muitos professores estão no grupo de risco para o desenvolvimento da doença (Abacar, Aliante \& António, 2020). Estes profissionais passam a vida cuidado das outras pessoas e, muitas vezes, não dispõe de tempo para cuidar de si mesmo e de seus colegas e quando percebem, já necessitam de ser cuidados (Carvalho, 2014).

A instituição deve estar atentada aos riscos do desenvolvido desta doença pelos profissionais e desenvolvidas manobras de enfrentamento com a finalidade de reduzir os problemas no ambiente de trabalho, minimizando as dificuldades, apoiando aos trabalhadores, propiciar-lhes boas condições de vida dentro e fora do ambiente de trabalho (Moreno, et al., 2010). Neste contexto, é importante que os profissionais construam e vivenciem um ambiente de apoio, de sustentação e suporte dentro da equipe, para que possam enfrentar e superar as tensões da prática profissional (Campos, 2011). O presente estudo realizou uma revisão integrativa com objetivo de identificar estratégias para a prevenção e tratamento da síndrome de Burnout, bem como analisar a resposta desta estratégia.

\section{Metodologia}

Para a realização desta revisão integrativa foi utilizado o método comparativo, do tipo quali-quantitativo, comparando os resultados da prevenção e tratamento entre os grupos tratados e não tratados (Pereira, et al., 2018). A pesquisa foi realizada no portal de periódicos Capes (Cesario, Carneiro \& Dolabela, 2020; Cruz, Almeida \& Dolabela, 2020), sendo utilizado o seguinte descritor- treatment and burnout.

Foram incluídos neste trabalho, somente os artigos que relatavam alguma medida de intervenção ou tratamento de burnout, no período de 1993 a 2021, cujo texto foi escrito em inglês, português ou espanhol. Como critério de exclusão foram adotados: excluído todo o material que não tivesse sido revisado pelos pares, em duplicidade, resumos, carta ao editor e comunicações rápidas. 
Após a exclusão dos materiais sem revisão pelos pares e de outros idiomas restaram 73 resumos para a leitura, então procedeu a exclusão dos resumos (6), carta ao editor (1), comunicação rápida (1), artigos em duplicidade ou fora do tema do trabalho (43). Todos os artigos selecionados foram lidos, sendo incluídos 18 artigos. O Fluxograma sintetiza as etapas metodológicas do trabalho (Figura 1).

Figura 1: Fluxograma de seleção de artigos.

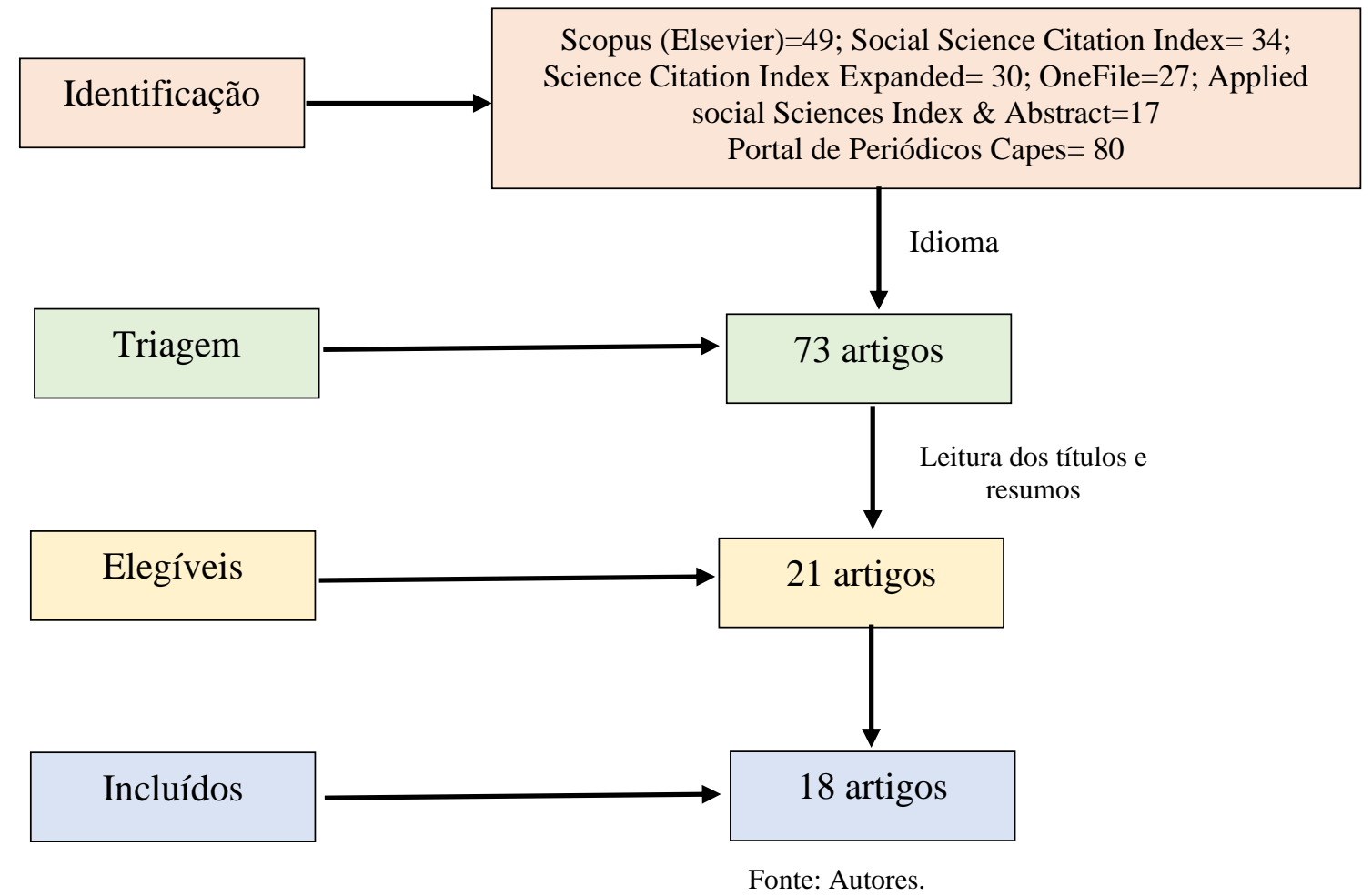

\section{Resultados e Discussão}

Após a exclusão dos artigos pelo idioma, iniciou-se a leitura dos títulos e resumos do material da triagem (73) e sempre que houve dúvida se o artigo tinha ou não relação com a pesquisa, este foi elegível. Então, realizou-se a leitura completa dos artigos e se confirmou a falta de relação com tema deste trabalho em 4 artigos elegíveis, sendo excluídos e ao final foram incluídos 17 estudos (Figura 1).

A maioria dos artigos incluídos teve como foco o tratamento da síndrome de burnout, enquanto que trabalhos prevenção desta doença ainda são minotários e são escassos estudos que avaliam o impacto de mudanças laborais e seu impacto na prevenção da doença (Tabela 1). Por isso, adota-se o risco de ter que afastar o profissional das atividades laborais para realizar o tratamento, ao invés de prevenir que este funcionário adoeça.

Refletindo sobre o processo saúde-doença percebe-se que a saúde é silenciosa e só percebemos sua perda quando adoecemos. No caso do Burnout e outros agravos é importante ouvir o próprio corpo para assegurar a saúde com qualidade, pois os limites entre a saúde e a doença são tênues. Vários fatores podem contribuir para o adoecimento, tais como: condições para execução das atividades, jornada de trabalho, satisfação profissional, relações profissionais, nível de estresse diário, entre outros (Alonso, 2014; Silva, et al., 2015). A identificação precoce destes fatores e adoção de medidas preventivas é muito importante, sendo adoção das medidas de prevenção importantes (Awa, Plaumann \& Walter, 2010). Traçar metas para prevenção dos agravos saúde é muito importante, pois a partir destas torna-se possível escolher a medidas preventivas a serem adotadas, estabelecendo o recurso necessário para sua implantação e também os recursos materiais (Evans, 1985; Tauil, 1998). 
Research, Society and Development, v. 10, n. 5, e11110514500, 2021

(CC BY 4.0) | ISSN 2525-3409 | DOI: http://dx.doi.org/10.33448/rsd-v10i5.14500

Tabela 1: Artigos incluídos no estudo.

\begin{tabular}{|c|c|c|}
\hline Artigos & Objetivos & Estratégias \\
\hline Meesters, Y \& Waslander, M. (2010). Burnout and Light Treatment. Stress and Health 26: 13-20 & Tratamento & Exposição a luz forte \\
\hline $\begin{array}{l}\text { Lennartssona, A.K., et al. (2016). Changes in DHEA-s levels during the first year of treatment in patients with } \\
\text { clinical burnout are related to health development. Biological Psychology, 120, 28-34 }\end{array}$ & Tratamento & Hormônio regenerativo S-DHEA \\
\hline $\begin{array}{l}\text { Daniels, A.H., et al. (2016). Orthopaedic Surgeon Burnout: Diagnosis, Treatment, and Prevention. Journal of the } \\
\text { American Academy of Orthopaedic Surgeons, 24, 4, 213-219 }\end{array}$ & Tratamento & $\begin{array}{l}\text { Atenção plena ou programas educacionais e } \\
\text { meditação }\end{array}$ \\
\hline $\begin{array}{l}\text { Anclair, M., et al. (2018). Cognitive behavioural therapy and mindfulness for stress and burnout: a waiting list } \\
\text { controlled pilot study comparing treatments for parents of children with chronic conditions. Scand J Caring Sci., 32, } \\
389-396\end{array}$ & Tratamento & Terapia Cognitiva Comportamental (TCC) \\
\hline $\begin{array}{l}\text { Haberthu, A.K., et al. (2009). Characterization of Patients Discharged From Inpatient Treatment for Burnout: Use of } \\
\text { Psychological Characteristics to Identify Aftercare Needs. Journal of Clinical Psychology, 65, 10, 1039-1055. }\end{array}$ & Tratamento & $\begin{array}{l}\text { TCC, técnicas de relaxamento, terapia corporal } \\
\text { e massagens, atividades esportivas, } \\
\text { psicofarmacoterapia e medicina complementar }\end{array}$ \\
\hline $\begin{array}{l}\text { Lacoursiere, R.B. (2001). "Burnout" and substance user treatment: the phenomenon and the administrator-clinician's } \\
\text { experience, Substance Use \& Misuse, 36:13, 1839-1874 }\end{array}$ & Tratamento & Coesão dos pares \\
\hline $\begin{array}{l}\text { Farber, B.A. (2000) Treatment Strategies for Different Types of Teacher Burnout. Psychotherapy in Practice, 56(5), } \\
675-689\end{array}$ & Tratamento & Psicoterapia \\
\hline $\begin{array}{l}\text { Gómez-Gascón, T., et al. (2013). Effectiveness of an intervention for prevention and treatment of burnout in primary health care } \\
\text { professionals. BMC Family Practice, } 14,173 \text {. }\end{array}$ & Tratamento & Diagnostico e workshop \\
\hline $\begin{array}{l}\text { Tzu, G, Bannerman, B \& Hill, N. (2016). From Grey Nothingness to Holistic Healing: A Non-Dual Approach to the } \\
\text { Treatment of Burnout. Int J Ment Health Addiction. }\end{array}$ & Tratamento & Holístico \\
\hline $\begin{array}{l}\text { Pallich, G., et al. (2020). Emotional competence predicts outcome of an inpatient treatment program for Burnout. } \\
\text { Journal of Affective Disorders. } 274,949-954\end{array}$ & Tratamento & Competência emocional (CE) \\
\hline $\begin{array}{l}\text { Itália, S., et al. (2008). Evaluation and art therapy treatment of the burnout syndrome in oncology units. Psycho- } \\
\text { Oncology, 17, 676-680 }\end{array}$ & Tratamento & Arteterapia \\
\hline $\begin{array}{l}\text { Litscher, G., et al. Improvement of the Dynamic Responses of Heart Rate Variability Patterns after Needle and Laser Acupuncture } \\
\text { Treatment in Patients with Burnout Syndrome: A Transcontinental Comparative Study. Evidence-Based Complementary and } \\
\text { Alternative Medicine, 2013, 1-6. }\end{array}$ & Tratamento & Acupuntura \\
\hline $\begin{array}{l}\text { Iserson, K.V. (2018). Burnout syndrome: global medicine volunteering as a possible treatment strategy. The Journal } \\
\text { of Emergency Medicine, Vol. } 54 \text {, No. 4, pp. } 516-521\end{array}$ & $\begin{array}{l}\text { Prevenção e } \\
\text { tratamento }\end{array}$ & 3 estratégias \\
\hline $\begin{array}{l}\text { Madsen, I.E.H. et al., (2015). Burnout as a risk factor for antidepressant treatment e a repeated measures time-to- } \\
\text { event analysis of } 2936 \text { Danish human service workers. Journal of Psychiatric Research } 65 \text { (2015) 47e52 }\end{array}$ & Aumento de risco & Antidepressivo \\
\hline $\begin{array}{l}\text { Oser,C.B., et al.(2013). Causes, Consequences, and Prevention of Burnout Among Substance Abuse Treatment } \\
\text { Counselors:A Rural Versus Urban Comparison. Journal of Psychoactive Drugs, } 45 \text { (1), 17-27 }\end{array}$ & Prevenção & $\begin{array}{l}\text { Apoio do colega de trabalho, supervisão clínica } \\
\text { e autocuidado }\end{array}$ \\
\hline $\begin{array}{l}\text { Warren, C.S., et al. (2012). A Qualitative Analysis of Job Burnout in Eating Disorder Treatment Providers. Eating } \\
\text { Disorders, 20:175-195 }\end{array}$ & Prevenção & Autocuidado \\
\hline $\begin{array}{l}\text { Moreno, F.N., et al. (2011). Estratégias e intervenções no enfrentamento da síndrome de Burnout. Rev. enferm. UERJ. 19(1), 140- } \\
\text { 5. }\end{array}$ & Prevenção & Ações variáveis \\
\hline
\end{tabular}

Legenda: S-DHEA: Sulfato de Dehidroepiandrosterona. Fonte: Autores (2021). 


\subsection{Estratégias para a prevenção da síndrome de Burnout}

A síndrome de burnout se desenvolve como consequência do estresse ocupacional prolongado (Maslach, et al., 2001; Borritz, 2006) podendo progride para depressão, sendo utilizados antidepressivos para o tratamento desta doença. É importante avaliar o impacto do uso de antidepressivos na síndrome de burnout, sendo observado assimetria na resposta entre homens e mulheres. As estimativas de risco absoluto mostraram que, para participantes com pontuação superior a 50 pontos na escala de burnout, o risco de entrar no tratamento com antidepressivos durante o período médio de acompanhamento de 3 anos foi de cerca de $10 \%$ nas mulheres e $18 \%$ nos homens. Esses números sugerem que os trabalhadores que experimentam altos níveis de burnout correm um risco substancial de desenvolver uma condição de saúde mental clinicamente significativa, podendo se aproxima de 1 em 5 participantes (Tabela 2). Esses resultados sublinham a importância de identificar métodos para prevenir o desenvolvimento de burnout em trabalhadores de serviços humanos (Madsen, et al., 2015).

A associação mais forte entre burnout e tratamento com antidepressivos em homens pode estar relacionada ao fato da forma diferente que estes lidam dom a doença (Galdas et al., 2005), que buscam ajuda em um estágio mais avançado do burnout do que as mulheres e, portanto, tenham maior probabilidade de desenvolver primeiro um tratamento que necessite de transtorno mental. As análises sugerem que a progressão e as consequências funcionais do burnout não são afetadas pelas condições psicossociais de trabalho, pelo menos não na coorte PUMA. Consequentemente, eles apontam para a necessidade de direcionar esforços preventivos para a prevenção primária do desenvolvimento de burnout ao invés de esforços direcionados para reduzir as consequências de esgotamento (Madsen, et al., 2015).

Em 2008, um estudo avaliou a síndrome de Burnout entre trabalhadores ruais e urbanos, sendo pesquisado se eles conheciam as causas, consequências e como prevenir. No entanto, houve várias diferenças entre os conselheiros rurais e urbanos em subtemas, com apenas conselheiros rurais citando políticas de escritório e baixo prestígio ocupacional como causas do esgotamento. Várias causas do esgotamento transcendem a localidade, de forma que tanto os conselheiros de abuso de substâncias rurais quanto urbanos lutam com uma variedade de fatores semelhantes (Tabela 2). Embora a retenção de clientes tenha sido expressa como uma preocupação entre os grupos de foco, os trabalhadores também conectaram o esgotamento à natureza crônica do vício e à fragilidade da recuperação de seus pacientes (Oser, et al., 2013).

Apesar das perguntas do grupo focal não indagassem especificamente sobre as três dimensões do esgotamento (ou seja, exaustão emocional, despersonalização e falta de realização pessoal), as evidências que emergiram da pesquisa do grupo focal sugerem que todos os três aspectos são relevantes para muitos dos os conselheiros trabalhando para fornecer serviços clínicos para clientes desafiadores. Conforme observado por todos os conselheiros, os clientes precisam de uma abordagem de tratamento holística que é difícil de entregar quando recursos limitados e fatores organizacionais, como carga de trabalho pesada e responsabilidades administrativas excessivas, podem fomentar a sensação de fadiga ocupacional (Oser, et al., 2013).

Outro estudo relatou que o grande número de casos de conselheiros com burnout estava relacionado com a baixa satisfação ocupacional (Broome, et al., 2009). O estudo incluído neste trabalho, o esgotamento foi associado a sobrecarga administrativa, a frustação relacionada a administração do serviço e questões políticas ao esgotamento dos conselheiros (Oser, et al., 2013), sendo estes problemas maior entre os trabalhadores rurais. Em geral, nos serviços rurais as instalações podem possuir um menor número de pessoal para fornecer serviços de tratamento (Bouffard \& Smith 2005), exigindo assim uma maior colaboração no local de trabalho e sobrecarga de trabalho (Oser, et al., 2013).

Vale destacar que esta síndrome tem impactos negativos para a pessoa, como financeiros e aposentadoria precoce. Também ocasiona problemas para o serviço, visto, elevar a taxa de absenteísmo e rotatividade de trabalhadores (Silva et al., 2015). Apesar de pouco discutida, a taxa desta doença, em especial entre os profissionais da saúde, é crescente (Khamisa, Peltzer \& Oldenburg, 2013; Dubois, et al., 2014), requerendo a implantação de medidas que visem a prevenção da doença. 
Muitas vezes, os próprios trabalhadores dão sugestões de estratégias para a prevenção da doença. Compreendem que devido uma convivência diária com os colegas, este podem reconhecer os sintomas (Oser, et al., 2013), sendo importante a realização de oficinas para capacitação destes trabalhadores. Outra questão importante sugerida pelos trabalhadores é a supervisão clínica como fator de proteção ao Burnout (Tabela 2) (Oser, et al., 2013). Muitas empresas ainda pensam que o estabelecimento de convênios com "planos de saúde", para que o trabalhador utilize quando estiver doente, é o suficiente. Entretanto, não compreende que a saúde do trabalho é o maior ativo de escola, do hospital e etc.. Diante disso, é importante que tenha uma infraestrutura para salvaguardas as condições físicas e mentais de seus trabalhadores, com objetivo de prevenção e promoção da saúde (Berger, 2020).

Além disso, os profissionais relatam a importância da conscientização dos administradores em relação ao problema e que este se preocupe com medidas de prevenção (Oser, et al., 2013). Entretanto os administradores estão inseridos em mundo capitalista e de acordo com Lara (2011): “o trabalho, no modo de produção capitalista, é determinado pelo processo de produção, no qual acidentar e adoecer são resultantes de relações sociais em que o trabalhador se torna apêndice da máquina. $\mathrm{O}$ trabalho que deveria gerar prazer, felicidade, na ordem do capital, causa fadiga, doenças, acidentes, sofrimentos físicos e mentais".

Outra questão muito importante levantada pelo trabalhado é o cuidado pessoal, que pode incluir meditação, tirar férias, reservar um tempo para conversar com um colega de trabalho ou apenas se envolver em outras tarefas além da terapia (Tabela 2) (Oser, et al., 2013). No Brasil, Reforma Trabalhista Brasileira acabou fragilizando as relações trabalhistas, potencializa a exposição aos riscos existentes no ambiente laboral, aumenta o risco de adoecimento, contrapondo-se à prevenção de agravos na saúde dos trabalhadores, também à proteção de sua saúde (Santana, et al., 2020) podendo impactar no cuidado pessoal do trabalhador. 
Tabela 2: Análise dos resultados das medidas utilizada para a prevenção de Burnout

\section{Intervenções}

Antipressivo $^{1}$

Apoio do colega de trabalho, supervisão clínica e autocuidado²

3 estratégias $^{3}$

Autocuidado $^{4}$

Ações variáveis

\section{Síntese dos resultados}

Burnout foi associado a um risco aumentado de tratamento com antidepressivos.

Relataram que os colegas de trabalho são capazes de reconhecer os sinais de Burnout. Estratégias de enfrentamento, incluindo um ouvido simpático e responsabilidade foram vistas como sendo necessárias. Também, há necessidade de supervisão clínica como fator de proteção ao Burnout. É importante que os administradores pensem sobre a questão e adotem medidas de prevenção. Cuidados pessoais incluiu meditação, tirar férias, reservar um tempo para conversar com um colega de trabalho ou apenas se envolver em outras tarefas além da terapia

$1^{\text {a }}$ tenta reduzir novos casos de burnout eliminando ou modificando os estressores do local de trabalho; $2^{\text {a }}$ ajudar os indivíduos a lidar de forma mais eficaz com situações de trabalho estressantes, em vez de diminuir os estressores do trabalho; $3^{\text {a }}$ tratar quem já sofre de burnout e também depende do aprendizado de mecanismos de enfrentamento mais eficazes. A maioria dos métodos atuais de prevenção e tratamento se concentra nos indivíduos, e as organizações de saúde provavelmente continuarão a se concentrar no tratamento dos médicos em vez de abordar os problemas subjacentes do sistema causal.

Examinou a) percpção do burnout, b) esforços para controlar o burnout e recomendações para evitar o Burnout. Características comuns de sujeitos com Burnout: patologia alimentar; características do paciente (por exemplo, conflito de personalidade); fatores relacionados ao trabalho (por exemplo, demandas de tempo); e questões financeiras (por exemplo, compensação inadequada). Para evitar o esgotamento, mais de $90 \%$ dos participantes se engajaram em comportamentos de autocuidado (por exemplo, exercícios, apoio social). Os profissionais em início de carreira foram encorajados a utilizar a supervisão, criar um equilíbrio entre vida e trabalho, se envolver no autocuidado e limitar o número de casos.

Três níveis de intervenções: estratégias organizacionais; estratégias individuais focadas nas respostas pessoais e estratégias combinadas centradas na interação do contexto ocupacional com o indivíduo. As ações preventivas não podem ser estigmatizadas como responsabilidade individual ou pelo relacionamento profissional-usuário, precisa ser trabalhada como um problema da relação indivíduo - processo de trabalho

Referências: 1-Madsen, et al., 2015; 2-Oser, et al., 2013; 3- Iserson, 2018; 4-Warren, et al., 2012, 5-Moreno, et al., 2011. Fonte: Dos autores. 
Pesquisas vem sendo desenvolvias com o objetivo de prevenir ou melhorar o esgotamento, podendo ser agrupadas em 3 grupos: tenta reduzir novos casos de burnout, eliminando ou modificando o local de trabalho estressores; segunda estratégia visa ajudar os indivíduos a lidar de forma mais eficaz com situações de trabalho estressantes, em vez de diminuir os estressores do trabalho; a terceira é tratar quem já sofre de burnout e também depende do aprendizado de mecanismos de enfrentamento mais eficazes (Tabela 2) (Iserson, 2018).

$\mathrm{Na}$ prática, entretanto, a maioria das estratégias de prevenção e tratamento de burnout presumem que a fonte do burnout é o funcionário individual, e não um ambiente de trabalho disfuncional. Neste contexto é importante incluir atividades para aumentar a autoconsciência dos profissionais e para melhorar sua resiliência e envolvimento com seu trabalho e profissão (National Academy of Medicine apud Iserson, 2018). Trabalhar a valorização do trabalho e a importância de sua realização, contribue para que as pessoas suportem uma maior carga de trabalho e se sintam recompensados por seus esforços (Maslach, Schaufeli \& Leiter, 2001).

As razões filosóficas e pragmáticas relacionadas ao foco no indivíduo, incluindo noções de causalidade e responsabilidade individual, são adotadas devido a suposição de que é mais fácil e mais barato mudar pessoas do que organizações (Maslach, 1998). Estratégias que demonstraram aumentar o investimento emocional de um indivíduo em seu trabalho, chamadas de reengajamento, podem ser o melhor método para prevenir o adoecimento (Maslach, 2017).

Outro estudo avaliou a percepção dos terapeutas em relação a síndrome de Burnout e percepção dos esforços que devem ser empreendidos para controlar a doença e recomendações para evitar. Os sujeitos incluídos com na pesquisa com a doença apresentavam algumas características em comum, tais como: patologia alimentar; características do paciente (por exemplo, conflito de personalidade); fatores relacionados ao trabalho (por exemplo, demandas de tempo); e questões financeiras (por exemplo, compensação inadequada). Para evitar o esgotamento, mais de $90 \%$ dos participantes se engajaram em comportamentos de autocuidado (por exemplo, exercícios, apoio social). Os profissionais em início de carreira foram encorajados a utilizar a supervisão, criar um equilíbrio entre vida e trabalho, se envolver no autocuidado e limitar o número de casos (Tabela 2) (Warren, et al., 2012).

Em relação ao tratamento da síndrome, $20 \%$ dos participantes, mais comumente sobre como manter limites, usar técnicas específicas consideradas úteis (por exemplos- psicoeducação e abordar as causas subjacentes) e manter um estilo particular na sessão (por exemplos- ser real e ser honesto e manter o senso de humor). A importância de trabalhar as questões pessoais foi apontada por $17 \%$ dos participantes, que aconselharam terapia pessoal, trabalhar as questões alimentares e de imagem corporal, e não tratar essa população se você tivesse histórico de transtorno alimentar. Além disso, $15 \%$ dos participantes aconselharam os colegas a se lembrarem de seu papel no tratamento desses pacientes, especialmente encorajando os médicos a não levar o trabalho para o lado pessoal ou a assumir responsabilidade excessiva pela mudança (Warren, et al., 2012). Em síntese, mais uma vez, o foco é no sujeito e não das causas que levaram estes.

Outro estudo propõe três níveis de intervenções para a prevenção desta síndrome: estratégias organizacionais; estratégias individuais focadas nas respostas pessoais e estratégias combinadas centradas na interação do contexto ocupacional com o indivíduo. As ações preventivas não podem ser estigmatizadas como responsabilidade individual ou pelo relacionamento profissional-usuário, precisa ser trabalhada como um problema da relação indivíduo - processo de trabalho (Tabela 2) (Moreno, et al., 2011).

Quando o programa é centrado no contexto ocupacional, este dá enfatize a necessidade de modificações na forma de desenvolvimento das atividades, no âmbito organizacional, tais como melhorar o ambiente trabalho, redução das situações estressantes e relações humanas (Garrosa, et al., 2002). Deste modo, a organização da maneira de trabalhar necessita ser revista, com o objetivo de promover o bem-estar e prevenir o surgimento de doenças, sendo importante a disponibilidade de recursos humanos e materiais suficientes, trabalhar a autonomia do funcionário, participação na tomada de decisão, chefias 
deve estar preparada para receber as reivindicações dos trabalhadores, avaliações periódicas do modo de produção, plano de desenvolvimento claro, respeitas as vocação dos funcionários e lotação no local que melhor se adapte, resolução de conflitos de forma imparcial e justa e incentivos salariais. Além da mudança empresarial, os profissionais precisam se adequar às novas formas de trabalho, sendo mais flexível para facilitar este processo (Moreno, et al., 2011).

Outra forma de olhar o problema é através do desenvolvimento de programas centrados na interação do contexto ocupacional e o indivíduo. Este tipo de estratégia compreende a doença como um resultado da relação do sujeito e o meio laboral. Desta forma integrada executa as modificações das condições de trabalho, bem como desenvolve trabalhos que visem modificar a percepção do trabalhador e o seu modo de enfrentamento em situações de estresse (Garrosa et al., 2002; Moreno, et al., 2011). Estudo já demonstrou que para a prevenção da síndrome de Burnout é importante estabelecer boas relações sociais no trabalho (Maslach \& Jackson, 1981) e ações preventivas como reuniões para discussões e reflexões dos problemas são importantes (Fernandes, et al., 2006; Santos, Oliveira \& Moreira, 2006; Moreno, et al., 2011). Também, dar ciência aos funcionários em relação ao risco de desenvolvimento da doença é importante (Carlotto \& Palazzo, 2006; Ferreira \& Martino, 2006) e contemplando ações em conjunto com a participação de todos (Carlotto \& Palazzo, 2006)

\subsection{Intervindo e tratando a síndrome de burnout}

Semelhante a prevenção, a maioria dos estudos que abordando a intervenção e/ou tratamento da síndrome de Burnout trabalharam com estratégias não medicamentosas, tais como psicoterapias e práticas alternativas complementares. Também, o foco principal foi o sujeito, da maior parte dos estudos, é o sujeito e não a instituição (Tabela 3). Permanecendo a lógica que mudar pessoas é mais fácil que a estrutura organizacional. De acordo com Hernandez e Caldas (2001) em relação a mudança empresarial: "Tanto a literatura acadêmica quanto a gerencial tendem a apontar a resistência à mudança - isto é, qualquer conduta que objetiva manter o status quo em face da pressão para modificá-lo - como uma das principais barreiras à mudança bem-sucedida".

Estudo investigou a eficácia da terapia cognitiva comportamental (TCC) e programa de atenção plena (mindfulness, FM) entre pais de crianças com patologias crônicas e estressado e com síndrome de burnout. Ambas intervenções diminuíram significativamente o estresse e o burnout e podem ser eficientes para reduzir os níveis de estresse e burnout em pais de crianças com doenças crônicas (Tabela 3) (Anclair, et al., 2017).

A razão pela qual a atenção plena (FM) parece afetar o estresse e burnout pode ser explicada pelo sistema de regulação emocional e pelo fato de que a atenção plena tem um impacto positivo no bem-estar (Gilbert, 2009; Flook, et al., 2013; Creswell \& Lindsay, 2014; Davidson, 2016; Wielgosz, et al., 2016). Em termos biológicos, diante de uma situação estressante sistema de alarme é ativado e sua função é fazer com que as pessoas busquem proteção. O treinamento da atenção plena ajuda a ativar o sistema calmante, que equilibra os pensamentos e emoções negativos e acalma o sistema de alarme (Gilbert, 2014). Isso resulta em pais acessando seus pontos fortes e estratégias de enfrentamento para lidar com as demandas e estressores (Anclair, et al., 2017).

Estudo demostrou uma associação entre burnout e baixa competência emocional (CE), definida como a habilidade de regular adequadamente a intensidade e expressão das emoções. Então, 113 pacientes psiquiátricos internados responderam questionários padronizados de autorrelato na entrada, alta e três meses após o tratamento, avaliando CE, esgotamento e depressão. Ao final do tratamento houve melhora na $\mathrm{CE}$, incluindo uma melhor regulação da emoção sendo observadas reduções no esgotamento e depressão. Este estudo apoia a CE como um mecanismo putativo de mudança no tratamento multimodal de pacientes com transtorno depressivo relacionado ao estresse e burnout (Tabela 3) (Pallich, et al., 2020).

Em termos de intervenções, tanto de grupo como individuais, a psicoterapia pode contribuir para o aumento dos níveis de competências emocionais. Em muitas das sessões individuais de psicoterapia, o processamento emocional deve ser o tópico 
central, sendo aumentar a competência emocional a meta central. Além disso, os pacientes receberam meditação mindfulness apresentaram melhora na resiliência a estímulos emocionais intensos (Aftanas e Golocheikine, 2001) e aumentou as emoções positivas (Amutio et al., 2015). Assim, o melhor reconhecimento das próprias emoç̃os e a regulação mais eficaz dos estados emocionais negativos ou desagradáveis, bem como a prática da atenção plena, podem ter contribuído para o aumento da $\mathrm{CE}$ nos pacientes (Pallich, et al., 2020). Treinamento especializado que visem melhorar as habilidades emocionais (Berking, 2015) com foco no relaxamento muscular, relaxamento respiratório, consciência não julgadora, aceitação e tolerância, autossuficiência compassiva, análise de emoções e modificação de emoções podem melhorar em um grau mais elevado, se o foco do tratamento na regulação da emoção for intensificado (Pallich, et al., 2020).

Outro estudo investigou como ex-pacientes de uma clínica de internação para terapia de burnout podem ser agrupados com base em suas características psicológicas e se esses grupos correspondem a diferentes níveis de sintomas residuais (depressão e burnout) e saúde mental geral. Quatro grupos específicos podem ser identificados (funcionais, disfuncionais, pragmáticos diretos, altruístas infelizes). Antes de considerar possíveis intervenções, seria útil avaliar individualmente em que medida a percepção as incongruências podem ser o produto de cognições disfuncionais (Tabela 3) (Haberthür, et al., 2009).

Os pensamentos disfuncionais desempenham um papel proeminente no funcionamento psicológico de um paciente, as intervenções cognitivas (Beck, 1995). Se desejos não realizados, a estratégias pode ser útil promover o desenvolvimento e / ou otimização de metas como parte de um treinamento de resolução de problemas (Vieth et al., 2003). Déficits na regulação da emoção, um treinamento direcionado de habilidades emocionais (Berking, 2007). estilo de enfrentamento principalmente orientado para a emoção, o tratamento de manutenção neste grupo poderia focar adicionalmente no aprimoramento das estratégias de enfrentamento orientadas para a tarefa (Haberthür, et al., 2009).

Treinamento de competências sociais com foco na capacidade de dizer não, pode ser indicado para este grupo. Como os altruístas infelizes relataram principalmente o uso de estratégias de enfrentamento orientadas para a emoção, o treinamento adicional de estratégias de enfrentamento mais ativas para estressores relacionados ao trabalho e de estratégias adaptativas para evitar o estresse pode beneficiar os altruístas infelizes. Os pragmáticos diretos relataram níveis ligeiramente elevados de incongruência e pareciam ter pequenos déficits em relação à regulação e compreensão de suas emoções. Portanto, também este grupo poderia se beneficiar do treinamento de competências selecionadas de regulação da emoção (por exemplo, compreensão das emoções) como parte dos cuidados de manutenção (Haberthür, et al., 2009).

Usando professores como um protótipo, observou-se três tipos de esgotamento: "desgaste", em que um indivíduo desiste, sentindo-se esgotado para enfrentar o estresse; Burnout "clássico", em que um indivíduo trabalha cada vez mais duro em face do estresse; e um tipo "subestimado", em que um indivíduo se depara não com graus excessivos de estresse per se (por exemplo, sobrecarga), mas sim com condições de trabalho monótonas e desestimulantes. Os principais argumentos apresentados são que: a) os médicos devem evitar tratar o esgotamento como se fosse um fenômeno único e adaptar seu tratamento ao tipo específico de burnout manifestado por seu cliente; b) esses tratamentos devem ser de natureza essencialmente integrativa. $\mathrm{O}$ insight psicanalítico, a reestruturação cognitiva, a preocupação empática e as técnicas de redução do estresse podem ser necessárias, embora em combinações diferentes, para tratar com sucesso o burnout de cada tipo (Farber, 2000).

De particular importância para o subgrupo esgotado de professores esgotados é a capacidade do terapeuta de ser empático e forjar alianças eficazes com todos os pacientes, demonstrando que realmente entende o que estão enfrentando ou quão pouco apoio ou apreciação estão recebendo. Estas condutas contribuem para o sucesso terapêutico e pode contribuir para que o professor adote perspectivas alternativas e ver o trabalho como uma oportunidade de sucesso, outros estressores normativos do desenvolvimento (por exemplo, a decisão de ter filhos) devem ser tratadas também (Farber, 2000). 
Metade de todos os cirurgiões ortopédicos apresenta sintomas de burnout, com as taxas mais altas relatadas em residentes e chefes de departamento de ortopedia. Objetivamente o burnout, os familiares e colegas devam estar cientes dos primeiros sinais de alerta e fatores de risco. Evidências emergentes indicam que intervenções baseadas em atenção plena ou programas educacionais combinados com meditação podem ser opções de tratamento eficazes (tabela 3) (Daniels, et al., 2016).

Atividades de apoio reduz a reatividade a experiências estressantes ou emocionalmente desafiadoras. sessões didáticas, bem como sessões de prática que se concentram na respiração e Hatha ioga (Goodman \& Schorling, 2012). Redução da exaustão emocional, despersonalização e elevação sentimentos de realização pessoal podem ser tratado com o programa educacional de 8 semanas focado na comunicação consciente para aumentar a autoconsciência, administrar conflitos e compreender o esgotamento e envolveram respiração e movimentos de ioga (Krasner, et al., 2009). Meta-análise sugere que o treinamento baseado em mindfulness é intervenção eficaz para o esgotamento (Regehr, et al., 2014) e estudo randomizado demonstrou oficinas de autocuidado por 2 meses melhora domínio de despersonalização (Martins, et al., 2011). Curso de curta duração ( 1 a 5 dias) de estratégias de enfrentamento e gerenciamento do estresse, melhorou consideravelmente o esgotamento emocional, que foi mantido por 0,3 anos (Isaksson Ro, et al., 2010). 
Research, Society and Development, v. 10, n. 5, e11110514500, 2021

(CC BY 4.0) | ISSN 2525-3409 | DOI: http://dx.doi.org/10.33448/rsd-v10i5.14500

Tabela 3: Análise dos resultados das medidas utilizadas para o tratamento da síndrome de Burnout.

\begin{tabular}{ll}
\hline Técnica & Síntese dos resultados \\
\hline TCC $^{1}$ & Tanto a TCC quanto intervenções de mindfulness foram eficazes melhorias significativas dos sintomas de burnout
\end{tabular}

Competência emocional $(\mathrm{CE})^{2} \quad 113$ pacientes psiquiátricos. O Questionário para a Autoavaliação da Competência Emocional foi usado para avaliar déficits e recursos na CE com nove subescalas: atenção, consciência das sensações corporais, clareza, compreensão, regulação, aceitação, resiliência, autossuficiência e prontidão para confrontar. Psicoterapia em grupo ou individuais contribui para o aumento dos níveis de CE, devendo ser focado na regulação da emoção, dando input teórico e pacientes engajados em exercícios práticos para melhorar a percepção e o tratamento das emoções. CE melhorado, incluindo uma melhor regulação da emoção durante o tratamento, previu resultados de tratamento favoráveis em relação aos sintomas de esgotamento e depressão.

Vários métodos

Quatro grupos específicos podem ser identificados (funcionais, disfuncionais, pragmáticos diretos, altruístas infelizes). Antes de considerar possíveis intervenções, seria útil avaliar individualmente em que medida a percepcão as incongruências podem ser o produto de cognições disfuncionais. Treinamento de competências sociais com foco na capacidade de

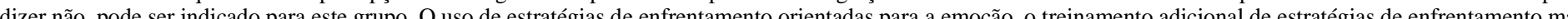
para estressores e de estratégias adaptativas para evitar o estresse pode beneficiar. Também, o treinamento de competências selecionadas de regulação da emoção (por exemplo, compreensão das emoções) como parte dos cuidados de manutenção.

Vários métodos ${ }^{4}$

Argumentos apresentados: a) os médicos devem evitar tratar o esgotamento dos professores como se fosse um fenômeno único e sim adaptar seu tratamento ao tipo específico de burnout manifestado por seu cliente; eb) esses tratamentos, embora incorporem elementos diferentes, devem ser de natureza integrativa. O insight psicanalítico, a reestruturação cognitiva, a preocupação empática e as técnicas de redução do estresse podem ser necessárias, embora em combinações diferentes, para tratar com sucesso o burnout de cada tipo Atenção plena ou programas Evidências emergentes indicam que intervenções baseadas em atenção plena ou programas educacionais combinados com meditação podem ser opções de tratamento eficazes educacionais e meditação Reestruturação Cognitiva Diagnóstico e workshop ${ }^{7}$

O insight psicanalítico, a reestruturação cognitiva, a preocupação empática e as técnicas de redução do estresse podem ser necessárias.

Este foi submetido a 16 horas de treinamento, concluindo que medidas organizacionais são importantes para prevenir a síndrome de burnout, mas também fornecer aos profissionais para lidar com estratégias, como esta intervenção em grupo pretende fazer

Holistico $^{8}$

O foco da vida deixou de ser espiritual e passou a ser o trabalho, onde de se espera realizações imediatas, sobrecarga de trabalho, individualismo e outras questões que levam a Burnout. A proposta de tratamento envolve: Psicologia existencial (logoterapia), Psicologia positiva, Perspectiva psicanalítica-existencial e Enfretamento da morte

Arteterapia $^{9}$

65 médicos e enfermeiras de um adulto (Grupo A) e uma unidade de oncologia pediátrica (Grupo B). Foi usado o Maslach Burnout Inventory para estimar o nível de Burnout e Grupo B foi submetido a um programa de intervenções de arteterapia. Avaliou o antes e depois da intervenção e houve redução do nível de burnout.

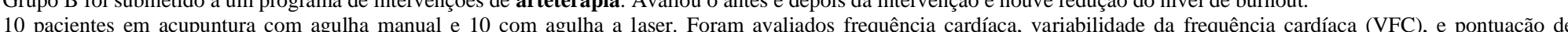

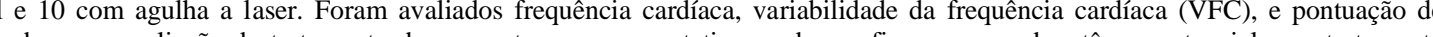
tratamento de acupuntura dinâmica (DATS). Com base na avaliação do tratamento de acupuntura neurovegetativa, pode-se afirmar que ambos têm o potencial para tratamento
complementar de pacientes com síndrome de burnout.

fratados com a luz; 6 estavam trabalhando, sendo 5 em meio período; 7 não estavam exercendo as atividades laborais, todos apresentavam condições clínicas similares. A melhoria após o tratamento com luz houve melhoria dos escores de exaustão emocional, porém não foi possível demonstrar nenhuma diferença significativa entre as duas condições com base nas outras pontuações de autoavaliação semanais.

Luz forte ${ }^{11}$

Coesão dos pares ${ }^{12}$

Aksamit, 1997- coesão dos pares, o apoio do supervisor, a autonomia em trabalho, ambiente de trabalho eficiente (orientação para tarefas), pressão de trabalho, clareza de regras e políticas e conforto físico no local de trabalho

Liu, 1997- estratégias de enfrentamento estão relacionadas ao "esgotamento" e são determinadas pelo que o ambiente de trabalho permitirá na autonomia dos membros da equipe para encontrar maneiras de fazer seu trabalho e no apoio do supervisor para o que é feito.

Hormônio Regenerativo S- 122 pacientes. Metade dos pacientes exibiram níveis aumentados de DHEA-S durante o ano, enquanto demais exibiu níveis diminuídos. Não houve diferença nos sintomas de DHEA $^{13}$ burnout ou estado de saúde associado no início do estudo entre os grupos. Maior reducão nos sintomas de burnout foram observados em pacientes com maiores níveis de DHEA-S. Além disso, os pacientes com níveis aumentados de DHEA-S tiveram melhor autoavaliação de saúde, vitalidade e bem-estar.

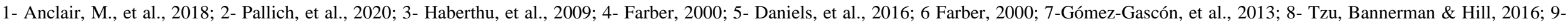

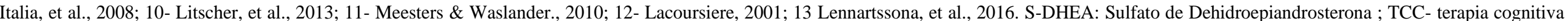
comportamental. Fonte: Autores. 
Trabalho descreveu um protocolo para um ensaio clínico controlado, pragmático e randomizado em 2 grupos paralelos: intervenção e controle. O diagnóstico foi feito através do inventário de burnout de Maslach, Questionário de Desgaste Professional Médico ou Questionário de Desgaste Professional de Enfermagem e Questionário de Saúde Geral, tendo um grupo controle e de intervenção. Este foi submetido a 16 horas de treinamento, como os seguintes tópicos: Sessão 1- A síndrome de burnout: identificando e enfrentando com os principais estressores do trabalho; Sessão 2- O processo de burnout: componentes cognitivos e motivacionais; Sessão 3- Fatores moderadores do processo de burnout: competência emocional e personalidade positiva; Sessão 4- Gerenciando as consequências do esgotamento: autocuidado e recuperação. Concluindo que medidas organizacionais são importantes para prevenir a síndrome de burnout, mas também fornecer aos profissionais para lidar com estratégias, como esta intervenção em grupo pretende fazer. Medidas organizacionais são importantes para prevenir a síndrome de burnout, mas também fornecer aos profissionais para lidar com estratégias, como esta intervenção grupal pretende fazer (Tabela 3) (Gómez-Gascón, et al., 2013).

Sabe-se que trabalho dos profissionais de saúde é altamente estressante e apresenta risco de esgotamento e a eliminação completa de alguns de seus gatilhos é praticamente impossível. No entanto, o efeito desses estressores pode ser moderado por recursos pessoais (Gálvez, Moreno \& Mingote, 2009; Gómez-Gascón, et al., 2013), é importante e fornecer às pessoas estratégias e ferramentas eficazes possam se adaptar às situações estressante (López, 2013).

A cultura ocidental, a ênfase no individualismo, juntamente com a falta de conexão entre as pessoas, restringiu a definição de si mesmo a se tornar enraizada em suas realizações (Schaufeli, et al., 2009), que buscam a satisfação egoísta e imediata, enquanto permanecem insatisfeitos com a busca, criou as condições ideais para o esgotamento (Lasch, 1979). O foco da vida deixou de ser espiritual e passou a ser o trabalho, onde de se espera realizações imediatas, sobrecarga de trabalho, individualismo e outras questões que levam a Burnout (Tabela 3) (Tzu, Bannerman \& Hill, 2016).

Para tentar resolver o esgotamento pode-se utilizar a psicologia positiva, que envolve um foco em traços individuais positivos e experiências subjetivas do passado, presente e futuro, a fim de melhorar a qualidade de vida e remover as visões de uma vida desolada e sem sentido. Há um foco em esperança, sabedoria, criatividade, visão do futuro, coragem, espiritualidade, responsabilidade e perseverança (Seligman \& Csikszentmihalyi 2000).

Outra técnica que deve ser adotada é a psicologia existencial, que se desenvolveu como uma tentativa de compreender o isolamento, a alienação e a falta de sentido na vida (Corey 2009). Frustrações existenciais se desenvolvem a partir da busca por uma existência significativa e podem se manifestar em preocupações espirituais (Frankl, 1984). Como tal, um logoterapeuta auxilia na jornada de construção de significado, criando consciência dos verdadeiros desejos dentro das profundezas de seu ser (Tzu, Bannerman \& Hill, 2016).

A técnica psicanalítico-existencialismo trabalha a criação de uma vida significativa e sentimentos de importância e significado. Na perspectiva psicanalítica-existencial é trabalhado a partir da noção de que a escolha da carreira se manifesta a partir de aspectos inconscientes que fornecem ímpeto para que se reconstitua e desfaça as experiências adversas da infância. Um estado de esgotamento pode ocorrer quando tais objetivos e expectativas inconscientes não são alcançados e, portanto, deixam de fornecer significado existencial na vida de alguém (Malach Pines, 2002).

Estudos sobre burnout nas relações de cuidado apontam para a importância de incorporar métodos psicológicos para o tratamento e prevenção do burnout. Psicoterapeutas australianos demonstraram que o bem-estar existencial, uma subescala dentro da Escala de Bem-Estar Espiritual (Ellison, 1983), atuou como uma salvaguarda para o efeito do trauma em exaustão emocional e que aqueles que relataram altos níveis de bem-estar existencial estavam mais bem equipados para evitar o esgotamento emocional, principalmente quando se trabalhava com clientes traumatizados (Hardiman \& Simmonds, 2013). 
Utilizando o inventario de Maslach foi estimado o nível de burnout nas dimensões de exaustão emocional, distanciamento (cognitivo e emocional) e redução da realização pessoal dos profissionais da saúde, sendo o nível médio-alto no Grupo A e nível médio-baixo no Grupo B. Na segunda parte do estudo, o Grupo B foi submetido a um programa de intervenções de arteterapia com o objetivo de reduzir o nível de burnout. A comparação das respostas dos participantes do Grupo B antes e depois da intervenção indicou uma diminuição estatisticamente significativa do nível de burnout (Tabela 3) (Italia, et al., 2008).

Quando se utilizou a arteterapia é importante ressaltar a participação da equipe, pelo menos $80 \%$ participaram de todas as reuniões e as viveram mais como uma atividade recreativa do que como uma atribuição de trabalho. Toda a intervenção foi composta por diferentes técnicas; infelizmente não fomos capazes de identificar claramente qual componente mais eficaz (Italia, et al., 2008).

Ainda foi investigado a acupuntura com agulha manual e com agulha laser como terapia complementar para pacientes com síndrome de burnout, sendo avaliado a frequência cardíaca, a variabilidade da frequência cardíaca (VFC) e uma nova pontuação chamada pontuação de tratamento de acupuntura dinâmica. O estudo documentou efeitos significativos na frequência cardíaca após o tratamento com acupuntura com agulha e efeitos significativos na VFC causados pela acupuntura com agulha e com agulha a laser. Com base nos novos escores de avaliação do tratamento de acupuntura neurovegetativa, pode-se afirmar que tanto a acupuntura não invasiva com agulha a laser quanto a acupuntura com agulha manual têm o potencial de ser uma abordagem poderosa para o tratamento complementar baseado em evidências de pacientes com síndrome de burnout (Litscher, et al., 2013).

O desenvolvimento de novas abordagens de tratamento da síndrome de burnout é importante, e a acupuntura invasiva (agulha) ou não invasiva (agulha laser) é uma possibilidade (Litscher, et al., 2013). Ensaio randomizado investigou a acupuntura para a síndrome da fadiga crônica, observando melhorias nas pontuações clínicas após a acupuntura simulada. Conclui-se que esses efeitos podem ser decorrentes da pressão das agulhas sham sobre os pontos de acupuntura, além do efeito do placebo (Ng \& Yiu, 2013).

Estudos com pessoas que sofrem de distúrbios afetivos sazonais e com pessoas saudáveis mostram que os níveis de energia podem ser melhorados expondo-os à luz forte. Em um pequeno estudo, foram pesquisados os efeitos do tratamento com luz em quem sofre de burnout, os níveis de energia dos pacientes melhoraram. Quando o tratamento com luz foi administrado antes de qualquer outro tratamento, postula-se que os resultados do segundo tratamento surtirão efeito mais rapidamente (Meesters \& Waslander, 2010). Outro estudo demonstrou que a exposição a luz diária por, pelo menos, 3 horas reduz o estresse e eleva a satisfação no trabalho, além disso, tem efeito indireto na prevenção de queixas de burnout (Alimoglu \& Donmez, 2005).

Profissionais envolvidos com o tratamento de usuários de drogas tendem a desenvolver a síndrome de bunout (Tabela 3) (Lacoursiere, 2001). Parece que o desenvolvimento da síndrome tem relação com os estressores profissionais e não profissionais (Price \& Spence, 1994) e que problemas domésticos contribuem para o esgotamento dos funcionários (Lacoursiere, 2001).

Uma estratégia para o enfrentamento do burnout é coesão dos pares, onde apoio do supervisor é importante, bem como a autonomia no trabalho, que o ambiente seja eficiente com orientações claras para as tarefas. Outros aspectos importantes são a redução da pressão no trabalho, clareza de regras e políticas e conforto físico no local de trabalho (Aksamit, 1997). As estratégias de enfrentamento em relação ao esgotamento são parcialmente determinadas pelo que é possível em determinado ambiente de trabalho (Tabela 3) (Liu, 1997). 
A partir da compreensão da condição de burnout, uma variedade de estratégias de tratamento ou enfrentamento podem ser adotadas. Às vezes, muitos fatores precisam ser tratados previamente ou concomitantemente, por exemplos, questões financeiras do funcionário, cuidar da vida doméstica, para reduzir o impacto da vida pessoal no esgotamento. As estratégias de prevenção para burnout incluem a detecção precoce pelos próprios funcionários e pelos administradores do programa, e um ambiente de trabalho que contribui para a realização pessoal sem fazer demandas excessivas (Lacoursiere, 2001).

As estratégias de tratamento pessoal incluem expectativas razoáveis para o resultado do paciente (Moos \& Moos,1998; Finney, Moos \& Timko, 1999), não trabalhando excessivamente, mas trabalhando de maneira mais inteligente, intervalos adequados do trabalho de alguns minutos para feriados mais longos e atendendo à vida não profissional e aos apoios. As estratégias organizacionais incluem ter uma equipe treinada, com habilidades de comunicação e ciente do paciente que irá atender e seus problemas (Lacoursiere, 2001).

Estudo avaliou o nível hormônio regenerativo DHEA-S e sua realação com o esgotamento clínico. Cerca de metade dos pacientes exibiu níveis aumentados de DHEA-S, enquanto a outra metade exibiu níveis diminuídos. Maior redução nos sintomas de burnout foi observada em pacientes nos quais os níveis de DHEA-S aumentaram durante o ano. Além disso, os pacientes com níveis aumentados de DHEA-S tiveram melhor autoavaliação de saúde, vitalidade e bem-estar (Lennartsson, et al., 2016).

O sulfato de eidroepiandrosterona (DHEA-S) é tem um papel regenerativo e protetor importante para a saúde (Maninger, et al., 2009; Theorell, 2009; Traish et al., 2011), pois possui propriedades antioxidantes, anti-inflamatórias e antiglicocorticóides e tem função na regeneração de células. A produção de DHEA-S atinge o pico na idade adulta jovem, posteriormente os níveis diminuem progressivamente em cerca de 2-4\% ao ano (Orentreich, et al., 1984). A redução de DHEA-S foi relatada como um marcador de deterioração da saúde, sendo que, os indivíduos mais velhos com menores níveis de DHEA-S foi $\mathrm{r}$ associado a maiores reduções na função cognitiva, bem como em várias medidas de fragilidade física

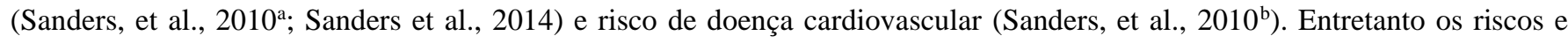
benefícios de sua reposição na síndrome de Burnout precisam ser avaliados.

\section{Conclusão}

Visando prevenir e tratar a síndrome de Burnout entre os trabalhadores é importante que tanto a empresa, quanto os funcionários compreenda a doença, os reais impactos das atividades laborais, bem como os aspectos da vida pessoal. Neste contexto, é importante mudanças na organização visando reduzir os fatores estressores, melhorando as relações humanas, a capacidade de diálogo entre a equipe, a autonomia profissional, entre outras questões.

Em relação a equipe de trabalho, cursos de capacitação que visem identificar os fatores estressores e elaborar estratégias de enfrentamentos são importantes. Neste contexto, um colega pode detectar o início da síndrome do colega e este deve ser encaminhado ao serviço de saúde. A coesão entre os pares, onde apoio do supervisor é importante, bem como a autonomia no trabalho, que o ambiente seja eficiente com orientações claras para as tarefas, redução da pressão no trabalho, clareza de regras e políticas, também pode ser uma estratégia interessante.

No campo individual é importante realizar um diagnóstico da gravidade do Burnout, os fatores estressores laborais e extra laborais e traçar um plano de tratamento individualizado. Este pode envolver atividades de autocuidado, psicoterapias, praticas alternativas complementares, bem como para cursos que visem auxiliar no enfrentamento dos fatores estressores. 
Research, Society and Development, v. 10, n. 5, e11110514500, 2021

(CC BY 4.0) | ISSN 2525-3409 | DOI: http://dx.doi.org/10.33448/rsd-v10i5.14500

\section{Referências}

Abacar, M., Aliante, G., \& António, F. (2020). Burnout em professores do ensino secundário. Research, Society and Development, 9,7 , e545973776. 10.33448/rsd-v9i7.3776.

Aftanas, L. I., \& Golocheikine, S. A. (2001). Human anterior and frontal midline theta and lower alpha reflect emotionally positive state and internalized attention: high-resolution EEG investigation of meditation. Neurosci. Lett., 310, 57-60. https://doi.org/ 10.1016/S0304-3940(01)02094-8.

Aksamit, P. (1997). Investigation of Burnout in Substance User Treatment Providers. Diss. Abst. Internat., 58, 4,1793B-1794B.

Alimoglu, M. K., \& Donmez, L. (2005). Daylight exposure and the other predictors of burnout among nurses in a university hospital. International Journal of Nursing Studies, 42, 549-555. 10.1016/j.ijnurstu.2004.09.001.

Alonso, F. G. (2014). Síndrome de burnout: manual de medidas preventivas e identificativas para aplicação pelo engenheiro de segurança do trabalho Monografia Curso de Pós-Graduação em Engenharia de Segurança do Trabalho, Universidade Tecnológica Federal do Paraná - UTFPR, 37p.

Amutio, A., Martínez-Taboada, C., Hermosilla, D., \& Delgado, L. C. (2015). Enhancing relaxation states and positive emotions in physicians through a mindfulness training program: a one-year study. Psychol. Health Med., 20, 720-731. https://doi.org/10. 1080/13548506.2014.986143.

Anclair, M., Lappalainen, R., Muotka, J., \& Hiltunen, A. J. (2018). Cognitive behavioural therapy and mindfulness for stress and burnout: a waiting list controlled pilot study comparing treatments for parents of children with chronic conditions. Scand J Caring Sci., 32, 389-396. 10.1111/scs.12473.

Awa, W. L., Plaumann, M., \& Walter, U. (2010). Burnout prevention: a review of intervention programs. Patient Education and Counseling, 78, 2, 184-190. 10.1016/j.pec.2009.04.008.

Beck, J.S. (1995). Cognitive therapy: Basics and beyond. Guilford.

Berger, A. (2020). Medicina Do Trabalho - Foco Na Prevenção E Promoção Da Saúde. Saúde Business. Https://www.saudebusiness.com/gesto/medicina-dotrabalho-foco-na-preveno-e-promoo-da-sade.

Berking, M. (2007). Training emotionaler Kompetenzen. [Training of emotional competences]. Berlin: Springer.

Berking, M. (2015). Training emotionaler Kompetenzen, (3a ed.), Psychotherapie: Praxis. Springer-Verlag, Berlin Heidelberg.

Borritz M. (2006). Burnout in human service work e causes and consequences. Copenhagen, Denmark: National Institute of Occupational Health, Denmark.

Bouffard, J. A., \& Smith, S. (2005). Programmatic, counselor and client-level comparison of rural versus urban drug court treatment. Substance Use and Misuse, 40, 3, 321-42. 10.1081/ja-200049340

Broome, K. M.; Knight, D. K.; Edwards, J. R. \& Flynn, P. M. (2009). Leadership, burnout, and job satisfaction in outpatient drug-free treatment programs. Journal of Substance Abuse Treatment, 37, 2, 160-70. 10.1016/j.jsat.2008.12.002.

Campos, E. P. (2011). Quem cuida do cuidador: uma proposta para os profissionais da saúde. Vozes, (4a ed,), 150 p.

Cândido, J., \& Rocha De Souza, L. (2017). Síndrome De Burnout: As Novas Formas De Trabalho Que Adoecem. Portal Dos Psicólogos, 12.

Carlotto, M. S., \& Palazzo, L. S. (2006). Síndrome de burnout e fatores associados: um estudo epidemiológico com professores. Cad. Saúde Pública, 22, 1017-6. Doi: https://doi.org/10.1590/S0102-311X2006000500014

Carvalho, V. A. (2014). Humanização e cuidados paliativos. Loyola ed.

Cesario, M. S. A., Carneiro, A. M. F., \& Dolabela, M. F. (2020). Tratamento medicamentoso em pacientes neonatos com cardiopatia congênita: apontamentos a partir da literatura recente. Research, Society and Development, 9, 11, e3339119946, 10.33448/rsd-v9i11.9946.

Corey, G. (2009). The theory and practice of counselling and psychotherapy. Belmont, (8a ed.), Brooks/Cole.

Creswell, J., \& Lindsay, E. (2014). How does mindfulness training affect health? A mindfulness stress buffering account. Curr. Dir. Psychol. Sci., 23, 401-7. https://doi.org/10.1177/0963721414547415.

Cruz, L. O., Almeida, M. K. C., \& Dolabela, M. F. (2020). Contribuições de terapias complementares e outras estratégias para o tratamento da esquizofrenia: uma revisão integrativa. Research, Society and Development, 9, 11, e76791110376. 10.33448/rsd-v9i11.10376.

Daniels, A. H., DePasse, J. M., \& Kamal, R. N. (2016). Orthopaedic Surgeon Burnout: Diagnosis, Treatment, and Prevention. Journal of the American Academy of Orthopaedic Surgeons, 24, 4, 213-219. http://dx.doi.org/10.5435/ JAAOS-D-15-00148.

Davidson, R. J. (2016). Mindfulness-based cognitive therapy and the prevention of depressive relapse: measures, mechanisms, and mediators. JAMA Psychiatry, 73, 547-8. 10.1001/jamapsychiatry.2016.0135.

Dubois, C. A., Bentein, K., Mansour, J. B., Gilbert, F., \& Bédard, J. L. (2014). Why some employees adopt or resist reorganization of work practices in health care: associations between perceived loss of resources, burnout, and attitudes to change. Int $J$ Environ Res Public Health, 11, 1,187-201. 10.3390/ijerph110100187.

Ellison, C. W. (1983). Spiritual well-being: Conceptualization and measurement. Journal of Psychology \& Theology, 11, 330-340. 
Research, Society and Development, v. 10, n. 5, e11110514500, 2021

(CC BY 4.0) | ISSN 2525-3409 | DOI: http://dx.doi.org/10.33448/rsd-v10i5.14500

Evans, A. S. (1985). The eradication of communicable diseases: myth or reality? American Journal of Epidemiology, 122,199-207.

Farber, B. A. (2000) Treatment Strategies for Different Types of Teacher Burnout. Psychotherapy in Practice, 56(5), 675-689. 10.1002/(sici)10974679(200005)56:5<675::aid-jclp8>3.0.co;2-d.

Fernandes, J. D., Melo, C. M. M., Gusmão, M. C. C. M., Fernandes, J., \& Guimarães, A. (2006). Saúde mental e trabalho: significados e limites de modelos teóricos. Rev Latino-Am Enfermagem, 14, 803-1.

Ferreira, L. C. R., \& Martino, M. M. F. (2006). O estresse do enfermeiro: análise das publicações sobre o tema. Rev. Cienc. Med., 15, 3, 241-8.

Finney, J. W., Moos, R. H., \& Timko, C. (1999). The Course of Treated and Untreated Substance Use Disorders: Remission and Resolution, Relapse and Mortality,In Addictions: a Comprehensive Guidebook, McCrady, B. S., Epstein, E. E. Eds.; Oxford University Press: 30-49.

Flook, L., Goldberg, S. B., Pinger, L., Bonus, K., \& Davidson, R. J. (2013). Mindfulness for teachers: a pilot study to assess effects on stress, burnout and teaching efficacy. Mind Brain Educ., 7, 182-195. 10.1111/mbe.12026.

Frankl, V. E. (1984). Man's search for meaning. Simon \& Schuster.

Galdas, P. M., Cheater, F., \& Marshall, P. (2005). Men and health help-seeking behaviour: literature review. J. Adv. Nurs., 49, 616e23. 10.1111/j.13652648.2004.03331.x.

Gálvez, M., Moreno, B., \& Mingote, J.C. (2009). El desgaste profesional del médico. Revisión y Guía de Buenas Prácticas. El vuelo de Ícaro. Díaz de Santos.

Garrosa, H. E., Benevides, P. A. M. T., Moreno, J. B., \& Gozalez, J. L. (2002). Prevenção e intervenção na síndrome de burnout: como prevenir (ou remediar) o processo de burnout. In: Benevides PAMT, organizador. Burnout: quando o trabalho ameaça o bem-estar do trabalhador. Editora Casa do Psicólogo, 224-67.

Gilbert, P. (2009). Introducing compassion focused therapy. Adv. Psychiatr. Treat., 15, 199-208. https://doi.org/10.1192/apt.bp.107.005264.

Gilbert, P. (2014). The origins and nature of compassion focused therapy. Br. J. Clin. Psychol., 53: 6-41. 10.1111/bjc.12043.

Gómez-Gascón, T., Martín-Fernández, J., Gálvez-Herrer, M., Tapias-Merino, E., Beamud-Lagos, M., Mingote-Adán, J. C., \& Grupo EDESPROAP-Madrid (2013). Effectiveness of an intervention for prevention and treatment of burnout in primary health care professionals. BMC Family Practice, $14,173$.

Goodman, M. J., \& Schorling, J. B. (2012). A mindfulness course decreases burnout and improves well-being among healthcare providers. Int. J. Psychiatry Med., 43, 2, 119-128. 10.2190/PM.43.2.b.

Haberthu, A. K., Elkuch, F. M., Holtforth, M. G., Hochstrasser, B., \& Soyka, M. (2009). Characterization of Patients Discharged From Inpatient Treatment for Burnout: Use of Psychological Characteristics to Identify Aftercare Needs. Journal of Clinical Psychology, 65, 10, 1039-1055. 10.1002/jclp.20606.

Hardiman, P., \& Simmonds, J. G. (2013). Spiritual well-being, burnout and trauma in counsellors and psychotherapists. Mental Health, Religion and Culture, 6, 1044-10. https://doi.org/10.1080/13674676.2012.732560.

Hernandez, J. M. C., \& Caldas, M. P. (2001). Resistência a mudança: uma revisão crítica. Rev. adm. empres., 41, 2, 31-45. http://dx.doi.org/10.1590/S003475902001000200004

Isaksson Ro, K. E., Tyssen, R., Hoffart, A., Sexton, H., Aasland, O. G., \& Gude, T. (2010). A three year cohort study of the relationships between coping, job stress and burnout after a counselling intervention for help seeking physicians. BMC Public Health, 10, 213.

Iserson, K. V. (2018). Burnout syndrome: global medicine volunteering as a possible treatment strategy. The Journal of Emergency Medicine, 54, 4, 516-521. doi: 10.1016/j.jemermed.2017.12.062.

Itália, S., Favara-Scacco, C., Di Cataldo, A., \& Russo, G. (2008). Evaluation and art therapy treatment of the burnout syndrome in oncology units. PsychoOncology, 17, 676-680. 10.1002/pon.1293

Khamisa, N., Peltzer, K., \& Oldenburg, B. (2013). Burnout in Relation to Specific Contributing Factors and Health Outcomes among Nurses: A Systematic Review. Int J Environ Res Public Health, 10, 6, 2214-40. https://doi.org/10.3390/ijerph10062214.

Kirby, E. E. F., Jung, I. E. F.de S., Neves, L. M. L., Gregório, A. P. A., \& Gouvêa, M. V. (2020). Síndrome de Burnout em profissionais que atuam na Oncologia: Revisão Integrativa. Research, Society and Development, 9, 7, e48973545. 10.33448/rsd-v9i7.3545.

Krasner, M. S., Epstein, R. M., Beckman, H., Suchman, A. L., Chapman, B., Mooney, C. J., \& Quill, T. E. (2009). Association of an educational program in mindful communication with burnout, empathy, and attitudes among primary care physicians. JAMA, 302, 12, 1284-1293. 10.1001/jama.2009.1384

Lacoursiere, R. B. (2001). "Burnout" and substance user treatment: the phenomenon and the administrator-clinician's experience, Substance Use \& Misuse, 36, 13 , 1839-74. http://dx.doi.org/10.1081/JA-100108430.

Lara, R. (2011). Saúde do trabalhador: considerações a partir da crítica da economia política. Rev. katálysis, 14, 1, 78-85. https://doi.org/10.1590/S141449802011000100009 .

Lasch, C. (1979). The culture of narcissism: American life in an age of diminishing expectations. Warner.

Lennartssona, A. K., Theorell, T., Kushnir, M. M., \& Jonsdottir, I. H. (2016). Changes in DHEA-s levels during the first year of treatment in patients with clinical burnout are related to health development. Biological Psychology, 120, 28-34. 
Research, Society and Development, v. 10, n. 5, e11110514500, 2021

(CC BY 4.0) | ISSN 2525-3409 | DOI: http://dx.doi.org/10.33448/rsd-v10i5.14500

Litscher, G., Liu, C. Z., Wang, L., Wang, L. P., Li, Q. Q., Shi, G. X., Gaischek, I., Litscher, D., \& Wang, X. M. (2013). Improvement of the Dynamic Responses of Heart Rate Variability Patterns after Needle and Laser Acupuncture Treatment in Patients with Burnout Syndrome: A Transcontinental Comparative Study. Evidence-Based Complementary and Alternative Medicine, 2013, 1-6. http://dx.doi.org/10.1155/2013/128721

Liu, H. (1997). Burnout and Organizational Commitment Among Staff of Publicly Funded Substance User Treatment Programs. Unpublished doctoral dissertation, University of Maryland, Baltimore.

López, A. V. (2013). Rasgos, trastornos de personalidad y comorbilidad psiquiátrica de profesionales sanitarios con Síndrome de Burnout (Tesis Doctoral). Madrid, España: Universidad Complutense de Madrid.

Madsen, I. E. H., Lange, T., Borritz, M., \& Rugulies, R. (2015). Burnout as a risk factor for antidepressant treatment e a repeated measures time-to-event analysis of 2936 Danish human service workers. Journal of Psychiatric Research, 65, 47e52. http://dx.doi.org/10.1016/j.jpsychires.2015.04.004 0022-3956

Malach Pines, A. (2002). A psychoanalytic-existential approach to burnout: demonstrated in the cases of a nurse, a teacher, and a manager. Psychotherapy: Theory, Research, Practice, Training, 39, 103-113. https://doi.org/10.1037/0033-3204.39.1.103.

Maninger, N., Wolkowitz, O. M., Reus, V. I., Epel, E. S., \& Mellon, S. H. (2009). Neurobiological and neuropsychiatric effects of dehydroepiandrosterone (DHEA) and DHEA sulfate (DHEAS). Frontiers in Neuroendocrinology, 30, 65-91. 10.1016/j.yfrne.2008.11.002.

Martins, A. E., Davenport, M. C., Del Valle, M. P., Lalla, S., Domínguez, P., Ormando, L., Ingratta, A., Gambarini, H., \& Ferrero, F. (2011). Impact of a brief intervention on the burnout levels of pediatric residents. J Pediatr., 87, 6, 493-498. 10.2223/JPED.2127

Maslach, C., \& Goldberg, J. (1998). Prevention of burnout: new perspectives. Appl Prev Psychol., 7, 63-74. http://dx.doi.org/10.1016/S0962-1849(98)80022$\mathrm{X}$.

Maslach, C., \& Jackson, S.E. (1981). The measurement of experienced burnout. Journal of Ocuppational Behavior, 2, 99-113. https://doi.org/10.1002/job.4030020205.

Maslach, C., Schaufeli, W.B., \& Leiter, M.P. (2001). Job burnout. Аnnи Rev Psychol., 52, 397-422. https://doi.org/10.1146/annurev.psych.52.1.397.

Maslach, C. (2017). Finding solutions to the problem of burnout. Consulting Psychol J Pract Res., 69, 143. http://dx.doi.org/10.1037/cpb0000090

Meesters, Y., \& Waslander, M. (2010). Burnout and Light Treatment. Stress and Health 26: 13-20. https://doi.org/10.1002/smi.1250.

Moos, R. H., \& Moos, B. S. (1998). The Staff Workplace and the Quality and Outcome of Substance User Treatment Treatment. J. Stud. Alcohol, 59, 43-51. 10.15288/jsa.1998.59.43.

Moreno, F. N., Gil, G. P., Haddad, M. C. L., \& Vannuchi, M. T. O. (2011). Estratégias e intervenções no enfrentamento da síndrome de Burnout. Rev. enferm. UERJ., 19, 1, 140-5.

National Academy of Medicine. Action collaborative on clinician well-being and resilience. https://nam.edu/initiatives/ clinician-resilience -and-well-being/.

Ng, S. M., \& Yiu, Y. M. (2013). Acupuncture for chronic fatigue syndrome: a randomized, sham-controlled trial with singleblinded design,” Alternative Therapies in Health and Medicine, 19, 4, 21-26.

Orentreich, N., Brind, J. L., Rizer, R. L., \& Vogelman, J. H. (1984). Age changes and sex differences in serum dehydroepiandrosterone sulfate concentrations throughout adulthood. The Journal of Clinical Endocrinology \& Metabolism, 59, 551-5. 10.1210/jcem-59-3-551.

Oser, C. B., Biebel, E. P., Pullen, E., \& Harp, K. L. H. (2013). Causes, Consequences, and Prevention of Burnout Among Substance Abuse Treatment Counselors: A Rural Versus Urban Comparison. Journal of Psychoactive Drugs, 45, 1, 17-27. 10.1080/02791072.2013.763558.

Pallich, G., Blättler, L., Penedo, J. M. G., Holtforth, M. G., \& Hochstrasser, B. (2020). Emotional competence predicts outcome of an inpatient treatment program for Burnout. Journal of Affective Disorders. 274,949-954.10.1016/j.jad.2020.05.139

Pereira, A. S., Shitsuka, D. M., Pereira, F. J., \& Shitsuka, R. (2018). Metodologia da pesquisa científica. UFSM, https://repositorio.ufsm.br/bitstream/handle/1/15824/Lic_Computacao_Metodologia-Pesquisa-Cientifica.pdf

Price, L, \& Spence, S. H. (1994). Burnout Symptoms amongst Drug and Alcohol Service Employees: Gender Differences in the Interaction Between Work and Home Stressors. Anxiety, Stress Coping, 7, 67-84. 10.1080/10615809408248394.

Regehr, C., Glancy, D., Pitts, A., \& LeBlanc, V. R. (2014). Interventions to reduce the consequences of stress in physicians: A review and metaanalysis. $J$. Nerv. Ment. Dis., 202, 5, 353-359. 10.1097/NMD.0000000000000130.

Rissardo, M. P., \& Gasparino, R. C. (2013). Exaustão emocional em enfermeiros de um hospital público. Esc Anna Nery Rev Enferm., 17, 1, 128-32. Doi: http://dx.doi.org/10.1590/S1414-81452013000100018.

Silva, S. C. P. S., Nunes, M. A, P., Santana, V. R., Reis, F. P., Machado-Neto, J., \& Lima, S. O. (2015). A Síndrome De Burnout Em Profissionais Da Rede De Atenção Primária À Saúde De Aracaju, Brasil. Ciênc. Saúde Colet., 20, 10, 3011-20. Https://Doi.Org/10.1590/1413-812320152010.19912014

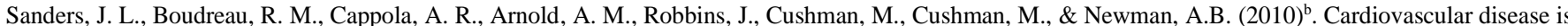
associated with greater incident dehydroepiandrosterone sulfate decline in the oldest old: The cardiovascular health study all stars study. Journal of the American Geriatrics Society, 58, 421-426. 10.1111/j.1532-5415.2010.02724.x. 
Research, Society and Development, v. 10, n. 5, e11110514500, 2021

(CC BY 4.0) | ISSN 2525-3409 | DOI: http://dx.doi.org/10.33448/rsd-v10i5.14500

Sanders, J. L., Cappola, A. R., Arnold, A. M., Boudreau, R. M., Chaves, P. H., Robbins, J., Cushman, M., \& Newman, A.B. (2010) a Concurrent change in dehydroepiandrosterone sulfate and functional performance in the oldest old: Results from the Cardiovascular Health Study All Stars study. The Journals of Gerontology. Series A, Biological Sciences and Medical Sciences, 65, 976-981. 10.1093/gerona/glq072.

Sanders, J. L., Ding, V., Arnold, A. M., Kaplan, R. C., Cappola, A. R., Kizer, J. R., Boudreau, R.M., Cushman, M., \& Newman, A.B. (2014). Do changes in circulating biomarkers track with each other and with functional changes in older adults? The Journals of Gerontology. Series A, Biological Sciences and Medical Sciences, 69, 174-181. 10.1093/gerona/glt088.

Santana, L. L., Sarquis, L. M. M., \& Miranda, F. M. D’ A. (2020). Riscos psicossociais e a saúde dos trabalhadores de saúde: reflexões sobre a Reforma Trabalhista Brasileira. Rev. Bras. Enferm. 73, 1, e20190092. https://doi.org/10.1590/0034-7167-2019-0092.

Santos, J. M., Oliveira, E. B., \& Moreira, A. C. (2006). Estresse, fator de risco para a saúde do enfermeiro em Centro de Terapia Intensiva. Rev enferm. UERJ., $14,580-5$.

Schaufeli, W. B., Leiter, M. P., \& Maslach, C. (2009). Burnout: 35 years of research and practice. Career Development International, 14, 204-220.

Sé, A. C. S., Machado, W. C. A., Passos, J. P., Gonçalves, R. C. S., Cruz, V. V., Bittencourt, L. P., Paiva, A. P. D. L.de, \& Figueiredo, N. M. A.de. (2020). Prevalência da síndrome de burnout em enfermeiros do atendimento pré-hospitalar. Research, Society and Development, 9, 7, e94097526510.33448/rsdv9i7.5265.

Seligman, M., \& Csikszentmihalyi, M. (2000). Positive psychology: An introduction. American Psychologist, 55, 5-14. https://doi.org/10.1037/0003066X.55.1.5

Tauil, P. L. (1998). Controle de agravos à saúde: consistência entre objetivos e medidas preventivas. Inf. Epidemiol. Sus, 7, 2, 55-58. http://dx.doi.org/10.5123/S0104-16731998000200006.

Teixeira, F. G., Silva, M. R. S. da, \& Medeiros, G. L. (2010). Síndrome de Burnout - a interface entre o trabalho na área da educação e na enfermagem. Revista de Enfermagem, serIII (2), 101-109.

Theorell, T. (2009). Anabolism and catabolism. In S. Sonnentag, P. L. Perrewé, \& D. C. Ganster (Eds.), Research in occupational stress and wellbeing. Current perspectives on job-stress recovery, 7, 249-276.

Traish, A. M., Kang, H. P., Saad, F., \& Guay, A. T. (2011). Dehydroepiandrosterone (DHEA)-a precursor steroid or an active hormone in human physiology. The Journal of Sexual Medicine, 8, 2960-82. 10.1111/j.1743-6109.2011.02523.x.

Tzu, G, Bannerman, B \& Hill, N. (2016). From Grey Nothingness to Holistic Healing: A Non-Dual Approach to the Treatment of Burnout. Int J Ment Health Addiction, 15, 652-69. 10.1007/s11469-016-9701-3.

Vieth, A. Z., Strauman, T. J., Kolden, G. G., Woods, T. E., Michels, J. L., \& Klein, M. H. (2003). Self-system therapy (SST): A theory-based psychotherapy for depression. Clinical Psychology: Science and Practice, 10, 245-68. https://doi.org/10.1093/clipsy.bpg023.

Warren, C. S., Schafer, K. J., Crowley, M. E., \& Olivardia, R. (2012). A Qualitative Analysis of Job Burnout in Eating Disorder Treatment Providers. Eating Disorders, 20,175-195. 10.1080/10640266.2012.668476.

Wielgosz, J., Schuyler, B., Lutz, A., \& Davidson, R. J. (2016). Long-term mindfulness training is associated with reliable differences in resting respiration rate. Sci Rep., 6, 27533. 10.1 